\title{
Investigating the community consequences of competition among clonal plants
}

\author{
LAURA GOUGH ${ }^{1 *}$, DEBORAH E. GOLDBERG ${ }^{2}$, CHAD HERSHOCK $^{2}$, \\ NIJOLE PAULIUKONIS ${ }^{1}$ and MARTINA PETRU ${ }^{3}$ \\ ${ }^{1}$ Department of Biological Sciences, University of Alabama, Tuscaloosa, AL 35487-0206, USA; \\ ${ }^{2}$ Department of Ecology and Evolutionary Biology, University of Michigan, Ann Arbor, MI 48109, \\ USA $;{ }^{3}$ Department of Botany, Faculty of Biological Sciences, University of South Bohemia, Braniso- \\ vksa 31, CZ-370 05, Ceske Budejovice, Czech Republic \\ (*author for correspondence, e-mail: lgough@biology.as.ua.edu)
}

Received 14 June 2001; accepted 5 December 2000

Co-ordinating editor: Brigitta Erschbamer

\begin{abstract}
Although clonal plants comprise most of the biomass of several widespread ecosystems, including many grasslands, wetlands, and tundra, our understanding of the effects of clonal attributes on community patterns and processes is weak. Here we present the conceptual basis for experiments focused on manipulating clonal attributes in a community context to determine how clonal characteristics affect interactions among plants at both the individual and community levels. All treatments are replicated at low and high density in a community density series to compare plant responses in environments of different competitive intensity. We examine clonal integration, the sharing of resources among ramets, by severing ramets from one another and comparing their response to ramets with intact connections. Ramet aggregation, the spacing of ramets relative to each other, is investigated by comparing species that differ in their natural aggregation (either clumped growth forms, with ramets tightly packed together, or runner growth forms, with ramets loosely spread) and by planting individual ramets of all species evenly spaced throughout a mesocosm. We illustrate how to test predictions to examine the influence of these two clonal traits on competitive interactions at the individual and community levels. To evaluate the effect of clonal integration on competition, we test two predictions: at the individual level, species with greater clonal integration will be better individual-level competitors, and at the community level, competition will cause a greater change in community composition when ramets are integrated (connected) than when they are not. For aggregation we test at the individual level: clumped growth forms are better competitors than runner growth forms because of their ability to resist invasion, and at the community level: competition will have a greater effect on community structure when ramets are evenly planted. An additional prediction connects the individual- and community-level effects of competition: resistance ability better predicts the effects of competition on relative abundance in a community than does invasion ability. We discuss additional experimental design considerations as revealed by our ongoing studies. Examining how clonal attributes affect both the individual- and community-level effects of competition requires new methods and metrics such as those presented here, and is vital to understanding the role of clonality in community structure of many ecosystems.
\end{abstract}

Key words: clonal integration, community, competition, ramet aggregation 


\section{Introduction}

Clonal plants dominate many grasslands, wetlands, and tundra, yet the influence of clonal characteristics on community and ecosystem processes is virtually unstudied. This preponderance of clonal growth forms in herbaceous communities may be caused by clonal attributes. For example, physiological integration among ramets or spatial arrangement of ramets within a clone may provide clonal plants with a competitive advantage through increased ability to acquire space horizontally via clonal growth, as well as by averaging out effects of neighborhood heterogeneity (e.g., Hartnett and Bazzaz, 1985). Clonal plants thus provide us with an important opportunity to link processes at different levels of organization, from individual physiology and morphology to community structure. To date, however, our actual knowledge of these linkages is limited by at least two factors. First, relatively few experiments directly address the community consequences of individual-level clonal attributes (Herben and Hara, 1997). Second, although many more studies have addressed the effect of clonal traits on components of individual performance, these have been conducted on a relatively small number of 'model' species (van Groenendael and De Kroon, 1990; de Kroon and van Groenendael, 1997).

Ecologists usually assume that the linkages between physiological and morphological traits of individuals and community level patterns operate at least partially through the influence of these traits on interspecific interactions such as competition. The prevailing assumption is that communities are structured in more complex ways than simply the collection of all species that can occur in a location in the absence of any interactions (a null community, sensu Zobel, 1992). However, the way in which species interactions translate individual traits into community structure requires consideration of two levels of comparison. Interactions must affect individuals and thus their growth, reproduction and survival. However, the intensity of these effects of competition on components of individual fitness does not necessarily predict the effect of competition at the level of the structure of the entire community, including the relative abundance of species. Community-level effects depend on the differences among species in their response to competition as well as on the absolute magnitude of these effects, thus we need specific indices that measure competition at the community level (Goldberg, 1994) as well as indices to assess individual-level competitive ability. Higher order interactions or indirect effects (e.g., Abrams, 1987) may additionally obscure the translation of patterns from individuals to communities. Consistent with these arguments, several studies have demonstrated that pairwise interactions focused on individuals may not predict community-level change (e.g., Silvertown et al., 1992).

We focus on two clonal attributes and their influence on both individualand community-level competitive ability: clonal integration, the sharing of 
resources such as water, sugar, and nutrients among sibling ramets, and clonal aggregation, the arrangement of ramets in space. We argue that to investigate competition among clonal plants we must take into account these specific aspects of clonal plant biology. Few authors have attempted to determine how these traits affect community-level processes (Herben and Hara, 1997), and they are not usually explicitly included in screening studies (e.g., Grime et al., 1990). In fact, many studies of interspecific interactions among clonal plants have used severed ramets or individual ramets grown from seed (e.g., Goldberg, 1987; Wilson and Tilman, 1993; Gaudet and Keddy, 1995). Such studies neglect the specific clonal attributes such as clonal integration that may confer an advantage or disadvantage to a particular species in a natural setting (Hartnett and Bazzaz, 1985; Pitelka and Ashmun, 1985). These studies also commonly neglect the spatial patterns of clonal plant growth which may influence community structure (Herben and Hara, 1997), limiting extrapolation from individual-level species responses to community-level consequences of species interactions. In addition, models of plant competition usually do not include clonal traits despite the likely correlation between clonal attributes and traits believed to determine competitive ability (e.g., Goldberg, 1990).

In this paper we develop predictions about how clonal aggregation and clonal integration influence competitive ability and community structure, and describe experiments to test these predictions. We also present hypothetical results that match our predictions to illustrate how these studies can be used to evaluate effects of clonal traits on species interactions, and how individual- and community-level competitive ability can be compared.

\section{Experimental approach}

Several different types of experiments need to be conducted to test the predictions outlined in the next sections. We focus our discussion on two common clonal growth forms: runners, with loosely spread ramets, and clumpers, with tightly packed ramets. First, an index of integration for the study species must be determined by conducting an integration experiment (e.g., those reviewed by Jónsdóttir and Watson, 1997). Second, to test the individual-level predictions, a pairwise competition experiment should be conducted. By quantifying the invasion (biomass) of a neighbor species into the target species' half of the pot, individual-level competitive ability can be measured for each species in a spatially explicit context as resistance and invasion ability. Rankings of species by competitive ability and level of integration can be compared among experiments with non-parametric tests of concordance (see Goldberg and Landa, 1991; Grace et al., 1993). Because both integration and pairwise experiments are frequently conducted (e.g., Goldberg and Landa, 1991; Silvertown et al., 
1992; Keddy et al., 1994), we will not discuss the methods involved further, but instead focus this discussion on the complementary community-level experiments.

\section{Community mesocosms}

To quantify both individual- and community-level effects of competition, we use the community density series design of Goldberg et al. (1995), where a mixture of species is grown in mesocosms both at low density, where species interactions are minimal, and at high density, where species interactions are plentiful. Because we expect greater variation among low density treatments, the low density mesocosms should be replicated more than the high density mesocosms. The magnitude of competition is quantified as the difference in some plant response between the low and high density environments.

In most competition experiments, the magnitude of competition is determined only for individual-level responses (e.g., growth or survival), which we term individual-level competitive ability. However, in formal ecological theory, competitive ability frequently refers to consequences of interactions for population dynamics or abundance rather than consequences for components of individual fitness (Laska and Wootton, 1998). Therefore, we also quantify the response to competition in terms of differences in relative population abundance between low and high density environments; we term this communitylevel competitive ability. For example, poor competitors are those that decline in relative abundance in high density mixture (species interactions intense) relative to low density mixtures (minimal or absent interspecific interactions), when abiotic conditions are held constant.

With the community mesocosm approach, both individual- and communitybased metrics can be examined in the same experiment to understand how interactions at the individual level scale up to patterns at the community level. Non-destructive data collected during the growing season can estimate individual-level responses, such as height per ramet (which can be used to estimate biomass with regression relationships established separately for each species) and per capita ramet production. The same data can be used to calculate community-level variables, in particular, relative abundance of each species in each treatment based on number of stems, number of ramets, or estimated biomass. At the conclusion of the experiment a destructive harvest should be conducted to investigate below ground relative abundance, as well as relative abundance among species based on total plant biomass.

To illustrate our predictions we construct a hypothetical dataset based on the relative competitive abilities of clumper and runner growth forms found in a community mesocosm experiment established in 1998 (Hershock and Goldberg, 2001; Hershock, unpublished data). We show how individual- and 
community-level competitive ability can be determined from a community density series of six hypothetical clonal, rhizomatous species, three runners and three clumpers, based on species used by Hershock. All clonal fragments (with equal number of ramets for all species) should be planted in their natural aggregation with ramet connections intact [connected-aggregated treatment (CA)], at both low and high densities.

We first plot the mean response in the low density mesocosms against the mean response in the high density mesocosms for each species in each treatment to evaluate the effect of the competitive environment. In the absence of density effects, performance in high and low density mesocosms should be the same, and the species should fall on the 1:1 line. Departure from the 1:1 line indicates the severity of competition. In the hypothetical example in Figure 1a (based on results from Hershock, unpublished), all species have per capita ramet production values on both axes greater than one, so on average all ramets produced offspring via clonal growth. However, this growth was suppressed in high density mesocosms for all species, so species fall below the 1:1 line, indicating they are negatively affected by competition. In this example, the runner species produced more ramets per capita than clumpers in the low density relative to the high, indicating the magnitude of growth suppression was greater for runners than clumpers (Fig. 1a).

At the community level, we show the runner species below and the clumper species above the 1:1 line in the CA treatment (Fig. 1b). The runner species increased in relative abundance in the low density environment, but increased less or even decreased in the high density environment, indicating they are poor community-level competitors (Fig. 1b). The clumpers decreased in relative abundance in the low density treatments, but displayed a slight increase or little change in the high density treatments, suggesting they are good communitylevel competitors.

To complement the graphical analysis and to quantify the difference in competitive ability among species, we calculate the $\ln \mathrm{RR}$ ( $\log$ response ratio) as:

$\ln \mathrm{RR}=\ln$ (response in high density/response in low density)

A value of zero for $\ln R R$ would indicate no density effect (therefore no effect of competitive environment), while negative values indicate poor competitive ability. When this ratio is calculated for the individual level in the CA treatment (from the data presented in Fig. 1a), mean values for both growth forms are negative, with the runners being poorer competitors than the clumpers (ln RR values are more negative; Fig. 2a). (Using actual results we would plot each species separately, but for ease of presentation we simply represent the hypothetical mean of the two growth forms.) At the community level, the better competitive ability of clumpers results in positive $\ln \mathrm{RR}$ values for this group, compared with negative values for runners (Fig. 2b). To analyze these 

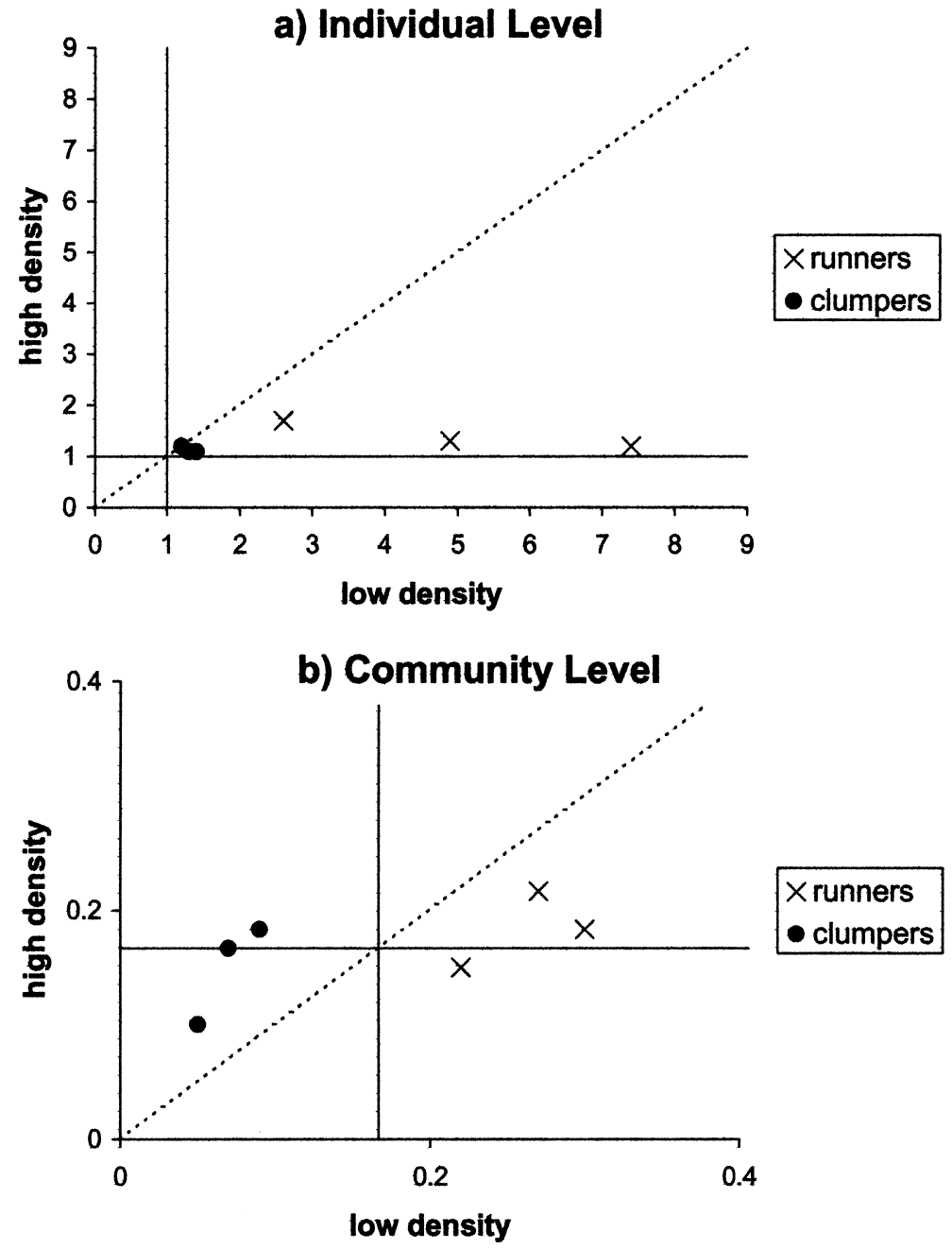

Figure 1. Hypothetical (a) individual-level and (b) community-level responses of three clumper and three runner species to competitive environment (density) in the connected-aggregated (CA) treatment after three growing seasons. Values plotted represent (a) per capita ramet production and (b) relative ramet abundance (percent) after two growing seasons. Solid lines represent (a) the baseline of growth to compare with new production and (b) the initial percent abundance $(17 \%$ or one-sixth of the community). The dashed line is the $1: 1$ line, indicating no effect of density on the response variables.

results statistically, we would perform a one-way ANOVA on the log response ratio with species as the main effect. Results of this analysis would reveal if individual species had significantly different competitive abilities.

Based on the differences in competitive ability between growth forms as illustrated here, we next describe specific predictions relating clonal integration 



Figure 2. Hypothetical mean log response ratios resulting from tests of integration by comparing CA (connected-aggregated) and SA (severed-aggregated) treatments after three growing seasons: (a) individual-level effects based on per capita ramet production and (b) community-level effects based on percent relative abundance. Positive values indicate good community-level competitors; negative values indicate poor community-level competitors. 
and ramet aggregation to competitive interactions. We employ a similar analysis as above, but incorporate experimental manipulations of the two clonal attributes to test these predictions, in addition to comparing among species that naturally differ in integration and aggregation. This dual approach avoids potentially confounding phylogenetic relationships among species that may hamper interpretation of responses between or among growth forms.

\section{Evaluating the role of clonal integration}

\section{Predictions}

An important aspect of clonal biology that should influence species interactions is the sharing of resources among ramets, i.e., clonal integration. Although clonal integration and ramet aggregation may be correlated, we treat them as separate attributes for the purposes of developing predictions and experimental treatments to tease apart their respective influence on interspecific interactions. Results to date indicate that physiological integration occurs for some period of time following new ramet production in most clonal plants (Hutchings and Bradbury, 1986) and is important for survival in heterogeneous environments (Price and Marshall, 1999). The concept of heterogeneity includes not only artificial light or soil resource environments, but the nature of the surrounding community, since plants themselves cause patchy resources (Huber-Sannwald et al., 1997). Integration in heterogeneous environments could affect competitive interactions in at least two important ways.

First, maternal transport of resources may make it easier for new ramets to become established in dense vegetation and therefore increase rates of horizontal spread compared to non-clonal species or clonal, but less integrated species (Wijesinghe and Handel, 1994; Stuefer et al., 1994). Thus, we predict that species with greater clonal integration will be better individual-level competitors, despite the physiological costs that may be associated with extensive integration (Jónsdóttir and Watson, 1997). For the purposes of this discussion, we assume that runners are better integrated than clumpers, based on preliminary results of our experiments.

The consequences of this correlation between competitive ability and integration ability for community-level attributes such as diversity are more complex. If the main cause of differential competitive ability among species is degree of integration, then removing integration (e.g., by severing connections) would make species more similar in competitive ability, and therefore decrease the rate of competitive exclusion and increase diversity (Huston, 1979; Agren and Fagerström, 1984; Shmida and Ellner, 1984). Although this seems the most likely scenario, the reverse situation could also arise: species that are poor 
competitors in the absence of integration could compensate by having high integration. In this case, removing integration from all species would increase the differences among species in net competitive ability and therefore increase competitive exclusion and decrease diversity, at least in the short term.

A second way in which clonal integration may affect community structure is by reducing the variance in competitive ability among individuals within a species, as well as affecting the mean differences among species in competitive ability, as described above. Variance within a species would be reduced because intragenet shoot density regulation occurs through a variety of means including integration (de Kroon, 1993), and because resource exchange among ramets within a genet should 'even out' the effects of small-scale heterogeneity in environmental conditions (Hartnett and Bazzaz, 1985; but see Hutchings and Price, 1993 for criticism of this study). If genets are distributed at random with respect to small-scale environmental heterogeneity, this should also mean that genets within a species would be more similar to each other on average. While the community consequences of reduced variation among individuals within a species in size and growth rate have not been modeled explicitly in clonal plants, we speculate that this reduced variation could decrease the potential for longterm coexistence, i.e., decrease diversity. This highly speculative hypothesis is based on the idea that increasing variance in size or growth rate within a species can allow coexistence of two species that differ in mean size, but that overlap considerably in the size distribution around that mean (Begon and Wall, 1987). Thus, removing integration should increase intraspecific variation in competitive ability (among ramets and genets) which should decrease interspecific variation in competitive ability and therefore increase diversity. We therefore predict that competition will cause less change in composition and diversity of communities consisting of genets with severed ramets than in communities of genets with connected ramets where integration is possible.

\section{Testing and evaluation}

To test these predictions relating clonal integration to competition, we employ the community density series as described above, and incorporate a treatment manipulating integration. In this severed-aggregated treatment (SA), rhizomes are first severed, then planted in their natural aggregation (same spatial arrangement as in the CA treatment). We expect the act of severing to decrease individual-level competitive ability of all species, but more severely for the runners, because we assume that runners rely more heavily on integration for successful new ramet production and survival than clumpers. Thus to meet our prediction that species with greater clonal integration are better individual-level competitors, when integration is removed (SA treatment), we expect runners to suffer from a larger decrease in competitive ability than clumpers, relative to 
the CA treatment (Fig. 2a). At the community level, removing integration should cause the species to become more similar to each other in competitive ability, thus reducing the effects of competition on the community, and causing the $\ln \mathrm{RR}$ values to get closer to zero for both growth forms (Fig. 2b). This result would meet our prediction of less community change caused by competition when ramets are not integrated compared to when they are connected. If the values of $\ln \mathrm{RR}$ statistically differ between CA and SA (as determined with a two-way ANOVA with species and treatment as main effects), integration affects competitive response of the study species; these differences at the level of individual species would be examined with post-hoc tests.

\section{Evaluating the role of clonal aggregation}

\section{Predictions}

Although many types of clonal architecture exist (Klimeš et al., 1997), we restrict our emphasis to two general classes of rhizomatous plants: clumped, with very short rhizome connections, and runner, with longer rhizomes between ramets (phalanx and guerilla, respectively, sensu Lovett Doust, 1981). Much clonal plant research has focused on these two clonal growth forms, and from this literature we make a tentative generalization: clumped growth forms are better at occupying and holding space than runner, but runner growth forms can invade newly opened space more rapidly than clumped (Schmid, 1985). This trade-off between these two growth forms has been found in experimental studies (Schmid and Harper, 1985; Cheplick, 1997; Humphrey and Pyke, 1998) and included in models of clonal plant growth (e.g., Bell, 1984; Herben, 1995; Winkler et al., 1999). This trade-off may result from the ability of runner species to send new ramets out at a greater distance from the parent ramet, thus acquiring more space per new ramet than clumpers, while clumper ramets are densely packed, constructing a difficult barrier to penetrate by other species. Expectations of how the degree of clonal aggregation affects individual-level competitive ability depend on whether competitive ability is defined as ability to invade and pre-empt unoccupied space (runners superior) or to resist invasion (clumpers superior). At the individual level, we therefore predict that species with a clumped growth form have higher resistance ability while species with a runner growth form have higher invasion ability, supporting the previously described trade-off. Therefore clumped growth forms should be better competitors than runner growth forms over time.

Predictions of how clonal aggregation affects community-level competitive ability are complicated by whether invasion or resistance ability is a more important process regulating overall abundance in the community. We expect 
that better resistance competitors will increase in relative abundance and dominate in more competitive (higher density) environments because of their ability to hold onto space. In contrast, we expect that runners will dominate in low density environments (e.g., early in succession) because of their advantage in exploring space. This advantage dissipates over time because of the growth pattern of runners where older ramets may die and give up the space they occupied, whereas clumped growth forms produce new ramets in such close proximity to older ramets they do not give up that space. In this way individual competitive ability, described above, can be directly related to competitive ability at the community level, as described below.

Degree of aggregation of ramets may affect diversity of entire communities by modifying the spatial distribution of interactions. Although not focused on aggregation of ramets in clonal plants, numerous models have suggested that coexistence is facilitated by intraspecific aggregation of a superior competitor (Atkinson and Shorrocks, 1981; Weiner and Conte, 1981; Pacala, 1986; Silvertown et al., 1992; Pacala and Levin, 1997). Intraspecific aggregation of ramets could therefore facilitate coexistence even under competitive conditions, i.e., decrease the overall impact of competition on species composition and maintain diversity of the community, even if individuals of different species that do come into contact interact very strongly. When clumpers (with greater ramet aggregation than runners) are competitively dominant, inferior competitors should be able to coexist, increasing diversity. In one of the only experimental tests of this idea with plants (four species that were not clonal), Stoll and Prati (2001) found that weaker competitors did in fact increase biomass when all species were aggregated, especially in high density environments, and that aggregation increased coexistence (see also Bergelson, 1990). We therefore predict that the experimental elimination of aggregation should decrease diversity by increasing the role of competition in structuring the community.

\section{Testing and evaluation}

The same community density series is used to examine the influence of aggregation on competitive ability, but with a different experimental manipulation replicated at low and high density. To test the effects of aggregation, mesocosms are established with ramets of all species evenly planted (severedeven, SE), and results are compared with the severed ramets planted in their natural aggregation (SA) as described earlier.

Using the same analysis as presented above, we examine effects of altering aggregation patterns. If our individual-level prediction is met, evenly planting individual ramets should result in decreased competitive ability for the runners after three growing seasons because interspecific interactions are more likely to occur, reducing average performance of runner ramets in high density envi- 




b) Community Level

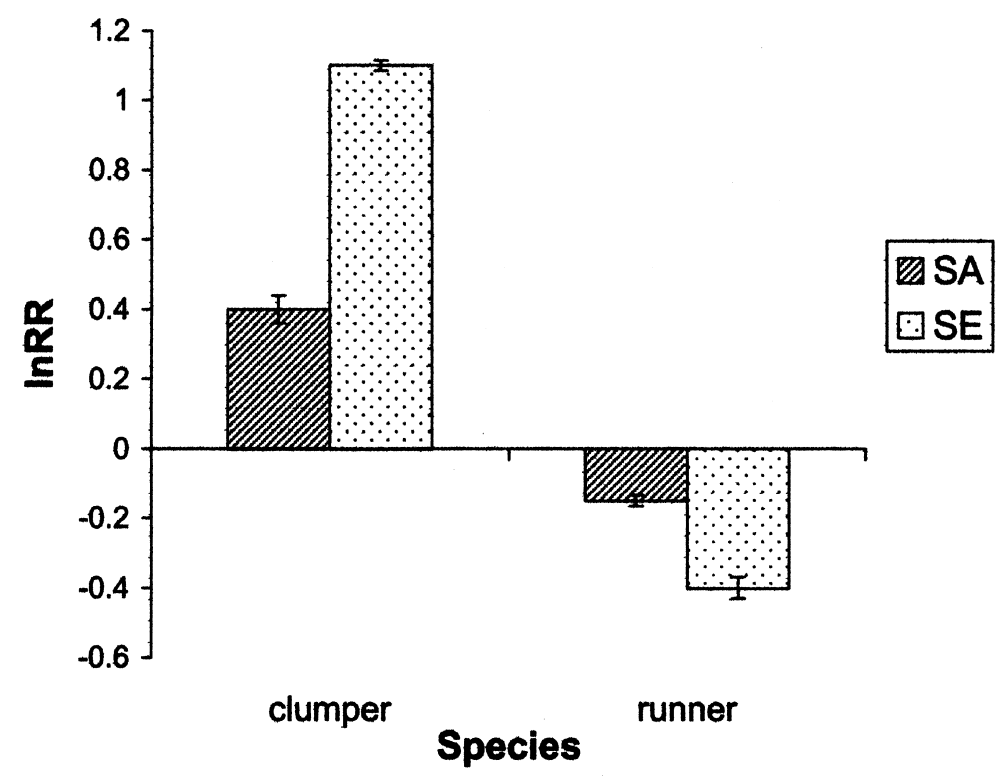

Figure 3. Hypothetical mean log response ratios resulting from tests of aggregation by comparing SA (severed-aggregated) and SE (severed-even) treatments after three growing seasons: (a) individual-level effects based on per capita ramet production, (b) community-level effects based on percent relative abundance. Positive values indicate good community-level competitors; negative values indicate poor community-level competitors.

[336] 
ronments (Fig. 3a). While runners may dominate initially at the community level because of higher horizontal growth rates, clumpers should better resist invasion over time as they 'clump'. We expect that removing natural aggregation patterns will cause competitive interactions to occur more frequently and thus cause more exclusion, exaggerating the differences between the two growth forms so that community-level competitive ability of clumpers increases while runners decrease (Fig. 3b). Consequently, we also expect the influence of competition on the community to be greater in SE than SA. Thus, values of ln RR for both growth forms should depart more from zero in SE than in SA.

\section{Additional analyses}

Other possibilities for analyzing effects of competition on community structure exist, such as diversity and evenness indices, and ordination techniques, e.g., principal component analysis. The rankings of species in competitive ability at the individual and community levels can be compared within the community mesocosm experiment. Results of pairwise competition studies can also be compared with results from the community-levels experiments to test if resistance ability better predicts the effects of competition on relative abundance in a community than invasion ability. For all analyses, results should be compared between clumper and runner species, and also within species for responses to altering natural integration and aggregation patterns.

\section{Experimental design issues}

We initiated a community density mesocosm experiment incorporating the three treatments described above in May 2000 using seven clonal, rhizomatous sedge species common to calcareous peatlands, known as fens, in southeastern Michigan (same species as Hershock used). We constructed wetland mesocosms mimicking soil and water chemistry and hydrology of natural fens in a garden at the Matthaei Botanical Gardens of the University of Michigan in Ann Arbor, Michigan, USA. We collected plants from various locations within and among fens in southeastern Michigan to randomize genotype within the treatments. Additional considerations have arisen as this study has progressed, and we discuss two of them below.

One important issue that must be considered in a community density design is that the low density treatments increase in plant density over time. As total ramet density and biomass increase, density in the initially low density mixture will approach that of the initially high density mixture. The magnitude of competition will increase in low density treatments through time, and species composition may converge between the two treatments. We are interested in 
the time scale of these trajectories, in particular how long it will take for the low density treatments to catch up to the density of the high density treatments, and how the communities compare at that time. In particular, natural fen community development is probably most similar to the trajectory of the low density CA treatment, and comparing this treatment to SA and SE should help us understand how clonal integration and ramet aggregation affect natural succession in this system. However, the predictions described here will be tested with measurements taken at the peak difference in species composition.

Another issue for consideration is how to maintain a severing treatment in a community-level experiment involving rhizomatous species. After one growing season, the high density mesocosms were completely filled with plants, and resevering connections in the severed treatments is impossible without drastically disturbing the soil and potentially killing many plants. For some of the species with relatively slow rates of new ramet production, this is not a significant problem, but for fast-growing species, the initial severing becomes less and less important over time as the clonal fragments increase in size.

\section{Important step in understanding competition among clonal plants}

Effects of competition among clonal plants need to be examined with a variety of approaches at both the individual and community levels to understand the impact of these interactions. Here we have presented predictions and experimental tests to illustrate one method to investigate these important phenomena. Several extensions of this work are obvious. One is to compare results in the mesocosms with growth and abundance responses in the field to determine how important competition is in structuring natural communities of these species. A second would be to incorporate a heterogeneity treatment into our community-level experiments; this would help place the results of the many population-level integration experiments conducted in artificially heterogeneous environments into a community context. Simultaneously examining clonal attributes and competition should lend important insights into the dynamics of clonal plant populations and communities as we continue to test our predictions of the effects of aggregation and integration on interspecific interactions at the individual and community level.

\section{Acknowledgements}

We thank many people for their help with establishing and collecting data from the community mesocosms including Zeena Monasa, Jeremy Parker, Tara Polosky, Duane Peltzer, Heidi Durbeck, Cindy Argue, Shannon Fiala, and 
Gabriel Tamaska, as well as the staff of the University of Michigan's Botanical Gardens for their continued, invaluable assistance, and the Michigan Department of Natural Resources for permission to collect plants on statemanaged land. T. Herben, I.S. Jónsdóttir, and J.F. Stuefer provided constructive comments on an earlier draft of this manuscript. This research was funded by a collaborative grant from the National Science Foundation (DEB99-74284 to L.G. and DEB99-74296 to D.E.G.). We are grateful to the organizers and participants of the Clone 2000 meeting for their insight into and support of these ideas.

\section{References}

Abrams, P.A. (1987) Indirect interactions between species that share a predator: varieties of indirect effects. In W.C. Kerfoot and A. Sih (eds) Predation: Direct and Indirect Impacts on Aquatic Communities. University Press of New England, Hanover, New Hampshire, pp. 38-54.

Ågren, G.I. and Fagerström, T. (1984) Limiting dissimilarity in plants: randomness prevents exclusion of species with similar competitive ability. Oikos 43, 369-375.

Atkinson, W.D. and Shorrocks, B. (1981) Competition on a divided and ephemeral resource: a simulation model. J. Anim. Ecol. 50, 461-471.

Begon, M. and Wall, R. (1987) Individual variation and competitor coexistence: a model. Func. Ecol. 1, 237-241.

Bell, A.D. (1984) Dynamic morphology: a contribution to plant population ecology. In R. Dirzo and J. Sarukhan (eds) Perspectives on Plant Population Ecology. Sinauer Associates, Inc., Sunderland, Massachusetts, pp. 48-65.

Bergelson, J. (1990) Life after death: site pre-emption by the remains of Poa annua. Ecology 71, 2157-2165.

Cheplick, G.P. (1997) Responses to severe competitive stress in a clonal plant: differences between genotypes. Oikos 79, 581-591.

de Kroon, H. (1993) Competition between shoots in stands of clonal plants. Plant Species Biol. 8, $85-94$.

de Kroon, H. and Van Groenendael, J. (eds) (1997) The Ecology and Evolution of Clonal Plants. Backhuys Publishers, Leiden.

Gaudet, C.L. and Keddy, P.A. (1995) Competitive performance and species distribution in shoreline plant communities: a comparative approach. Ecology 76, 280-291.

Goldberg, D.E. (1987) Neighborhood competition in an old-field plant community. Ecology 68 , 1211-1223.

Goldberg, D.E. (1990) Components of resource competition in plant communities. In J.B. Grace and D. Tilman (eds) Perspectives in Plant Competition. Academic Press, San Diego, California, pp. 27-49.

Goldberg, D.E. (1994) On testing the importance of competition for community structure. Ecology 75, 1503-1506.

Goldberg, D.E. and Landa, K. (1991) Competitive effect and response: hierarchies and correlated traits in the early stages of competition. J. Ecol. 79, 1013-1030.

Goldberg, D.E., Turkington, R. and Olsvig-Whittaker, L. (1995) Quantifying the community-level effects of competition. Folia Geobotanica and Phytotaxonomica 30, 231-242.

Grace, J.B., Guntenspergen, G.R. and Keough, J. (1993) The examination of a competitive matrix for transitivity and intransitive loops. Oikos 68, 91-98.

Grime, J.P., Hodgson, J.G. and Hunt, R. (1990) The Abridged Comparative Plant Ecology. Unwin Hyman, Boston. 
Hartnett, D.C. and Bazzaz, F.A. (1985) The integration of neighborhood effects by clonal genets of Solidago canadensis. J. Ecol. 73, 415-428.

Herben, T.H. (1995) Founder and dominance control: neglected concepts in the community dynamics of clonal plants. Abst. Bot. 19, 3-10.

Herben, T. and Hara, T. (1997) Competition and spatial dynamics of clonal plants. In H. de Kroon and J. van Groenendael (eds) The Ecology and Evolution of Clonal Plants. Backhuys Publishers, Leiden, pp. 331-358.

Hershock, C. and Goldberg, D.E. (2001) Community-level consequences of competition among clonal sedges. Abstract of the 86th Annual Meeting of the Ecological Society of America, August 5-10, 2001, Madison, Wisconsin.

Huber-Sannwald, E., Pyke, D.A. and Caldwell, M.M. (1997) Perception of neighboring plants by rhizomes and roots: morphological manifestations of a clonal plant. Can. J. Bot. 75, 2146-2157.

Humphrey, L.D. and Pyke, D.A. (1998) Demographic and growth responses of a guerrilla and a phalanx perennial grass in competitive mixtures. J. Ecol. 86, 854-865.

Huston, M. (1979) A general hypothesis of species diversity. Am. Nat. 113, 81-101.

Hutchings, M.J. and Bradbury, I.K. (1986) Ecological perspectives on clonal perennial herbs. BioScience 36, 178-182.

Hutchings, M.J. and Price, E.A.C. (1993) Does physiological integration enable clonal herbs to integrate the effects of environmental heterogeneity? Plant Species Biol. 8, 95-105.

Jónsdóttir, I.S. and Watson, M.A. (1997) Extensive physiological integration: an adaptive trait in resource-poor environments? In H. de Kroon and J. van Groenendael (eds) The Ecology and Evolution of Clonal Plants. Backhuys Publishers, Leiden, pp. 109-136.

Keddy, P.A., Twolan-Strutt, L. and Wisheu, I.C. (1994) Competitive effect and response rankings in 20 wetland plants: are they consistent across three environments? J. Ecol. 82, 635-643.

Klimeš, L., Klimešová, J., Hedriks, R. and van Groenendael, J. (1997) Clonal plant architecture: a comparative analysis of form and function. In H. de Kroon and J. van Groenendael (eds) The Ecology and Evolution of Clonal Plants. Backhuys Publishers, Leiden, pp. 1-30.

Laska, M.S. and Wootton, J.T. (1998) Theoretical concepts and empirical approaches to measuring interaction strength. Ecology 79, 461-476.

Lovett Doust, L. (1981) Population dynamics and local specialization in a clonal perennial ( $R a$ nunculus repens). I. The dynamics of ramets in contrasting habitats. J. Ecol. 69, 743-756.

Pacala, S.W. (1986) Neighborhood models of plant population dynamics. 2. Multispecies models of annuals. Theor. Popul. Biol. 29, 262-292.

Pacala, S.W. and Levin, S.A. (1997) Biologically generated spatial pattern and the coexistence of competing species. In D. Tilman and P. Kareiva (eds) Spatial Ecology. Princeton University Press, Princeton, NJ, pp. 204-232.

Pitelka, L.F. and Ashmun, J.W. (1985) Physiology and integration of ramets in clonal plants. In J.B.C. Jackson, L.W. Buss and R.E. Cook (eds) Population Biology and Evolution of Clonal Organisms Yale University Press, New Haven, pp. 399-443.

Price, E.A.C. and Marshall, C. (1999) Clonal plants and environmental heterogeneity. Plant Ecol. 141, 3-7.

Schmid, B. (1985) Clonal growth in grassland perennials II. Growth form and fine-scale colonizing ability. J. Ecol. 73, 809-818.

Schmid, B. and Harper, J.L. (1985) Clonal growth in grassland perennials I. Density and patterndependent competition between plants with different growth forms. J. Ecol. 73, 793-808.

Shmida, A. and Ellner, S. (1984) Coexistence of plant species with similar niches. Vegetatio 58, 29-55.

Silvertown, J., Holtier, S., Johnson, J. and Dale, P. (1992) Cellular automaton models of interspecific competition for space - the effect of pattern on process. J. Ecol. 80, 527-534.

Stoll, P. and Prati, D. (2001) Intraspecific aggregation alters competitive interactions in experimental plant communities. Ecology 82, 319-327.

Stuefer, J.F., During, H.J. and de Kroon, H. (1994) High benefits of clonal integration in two stoloniferous species, in response to heterogeneous light environments. J. Ecol. 82, 511-518.

van Groenendael, J.M. and de Kroon, H. (eds) (1990) Clonal Growth in Plants: Regulation and Function. SPB Academic Publishing, The Hague. 
Weiner, J. and Conte, P.T. (1981) Dispersal and neighborhood effects in an annual plant community. Ecol. Model. 13, 131-147.

Wijesinghe, D.K. and Handel, S.N. (1994) Advantages of clonal growth in heterogeneous habitats: an experiment with Potentilla simplex. J. Ecol. 82, 495-502.

Wilson, S.D. and Tilman, D. (1993) Plant competition and resource availability in response to disturbance and fertilization. Ecology 74, 599-611.

Winkler, E., Fischer, M. and Schmid, B. (1999) Modelling the competitiveness of clonal plants by complementary analytical and simulation approaches. Oikos 85, 217-233.

Zobel, M. (1992) Plant species coexistence - the role of historical, evolutionary and ecological factors. Oikos 65: 314-320. 